\title{
Philosophiques
}

\section{Philosophie et culture générale}

\section{Michel Bédard}

Volume 5, numéro 2, octobre 1978

URI : https://id.erudit.org/iderudit/203105ar

DOI : https://doi.org/10.7202/203105ar

Aller au sommaire du numéro

Éditeur(s)

Société de philosophie du Québec

ISSN

0316-2923 (imprimé)

1492-1391 (numérique)

Découvrir la revue

Citer ce document

Bédard, M. (1978). Philosophie et culture générale. Philosophiques, 5(2),

381-396. https://doi.org/10.7202/203105ar d'utilisation que vous pouvez consulter en ligne.

https://apropos.erudit.org/fr/usagers/politique-dutilisation/ 


\title{
INTERVENTIONS
}

\section{LE PROBLÈME DE L'ENSEIGNEMENT DE LA PHILOSOPHIE : DU CÔTÉ DES POURQUOI}

\section{PHILOSOPHIE ET CULTURE GÉNÉRALE}

\author{
par Michel Bédard
}

\author{
L'éducation peut tout. (HELVÉTIUS, \\ De l'bomme, de ses facultés intellectuelles et \\ de son éducation, $\mathrm{X}$.) \\ Or, la culture ne se transmet \\ point ( . . .). (ALAIN, Propos sur l'édu- \\ cation, XLV.)
}

\section{LE PROBLÈME}

L'enseignement de la philosophie au niveau préuniversitaire soulève des questions fort complexes que, trop souvent, on ne traite pas adéquatement par défaut de situer le débat dans un contexte assez large pour que chacune de ses facettes accuse son relief propre. Ainsi, les analyses du statut et du rôle de l'enseignement de la philosophie en regard de ce qu'il est convenu d'appeler la culture générale tiennent fréquemment pour acquise une définition de cette dernière et, dès lors, s'attaquent immédiatement à la signification du cours de philosophie dans le processus global de l'éducation. Or, une telle pratique n'a guère pour conséquence que d'occulter l'un des pôles essentiels du problème et, par là, de tronquer une question dont la solution - si tant est qu'il y en ait une devient toujours plus évanescente. Cela étant, il convient de tenter ici une étude du problème qui, malgré les limites qu'on lui a imparties, n'esquive aucun de ses aspects. 
Pour autant, toutefois, il ne faudrait pas croire que la difficulté sera tranchée. En effet, les relations entre philosophie et culture générale ont, toujours et partout, été problématiques, et ce, pour des raisons tenant vraisemblablement à leur nature même. Qu'on se remémore ici les différends qui opposèrent Isocrate à Platon, les durs reproches qu'Épicure adressa aux études libérales d'inspiration aristotélicienne, et l'on verra jusqu'où remonte le problème! Qu'on songe, par ailleurs, à la réforme Haby, en France, ou encore au « core curriculum » récemment mis de l'avant par l'université Harvard, et l'on verra jusqu'où s'étend ce même problème ! La question n'est donc ni d'ici ni de maintenant. Et ce phénomène ne devra jamais être perdu de vue puisque aussi bien ailleurs qu'autrefois les solutions proposées ont notablement varié, toutes ayant leur valeur et chacune prêtant flanc à la critique. Par conséquent, il n'y a pas lieu de chercher, en ce domaine, une solution apodictique: au vrai, l'élaboration d'une problématique consistante y représente déjà un gain notable.

Qu'est donc la culture générale? A-t-elle sa place en éducation? Et quelle contribution, le cas échéant, l'enseignement de la philosophie peut-il lui fournir?

\section{LA NOTION DE CULTURE GÉNÉRALE}

Le concept de culture générale est tributaire d'une histoire qui va du pepaideumenos antique jusqu’à l'homme cultivé contemporain, en passant notamment par l'humaniste de la Renaissance et l'honnête homme du XVII ${ }^{e}$ siècle. Sans relater par le menu cet historique qui serait pourtant précieux à l'intelligence de la notion, il importe de remarquer combien il met en évidence tout à la fois la permanence d'un certain souci et l'inconstance des modalités qu'il revêt suivant les époques et les milieux. Or, l'on ne doit pas confondre celles-ci avec celui-là sous peine de concevoir la culture générale de façon inacceptable, voire ridicule.

Dans cette perspective, deux idées, hélas ! assez répandues, de la culture générale semblent décidément à rejeter. Premièrement, celle qui, à tout prendre, la réduit à quelque émaillage 
dont les salonnards puissent faire étalage pour la galerie. On retrouve ici les thèses que font valoir les tenants du vernis culturel dont le sens de l'apparat, certes remarquable, ne compense point cependant la fragilité épidermique d'une position qui retire à la mémoire son statut ancillaire pour lui conférer, aux dépens de l'intelligence, des qualités de pénétration qu'elle ne peut assumer. En deuxième lieu, celle qui, plus subtilement, la fait consister dans la multiplicité des informations comprises. Dans ce cas, on constate, bien sûr, une certaine réhabilitation de l'intelligence, mais d'une intelligence perdue dans un encyclopédisme de mauvais aloi, d'une intelligence inapte à dominer l'érudition, d'une intelligence handicapée somme toute à raison de son incapacité de remplir sa fonction synthétique. Il appert en conséquence que ces deux conceptions ne coïncident pas avec cet idéal que recouvre confusément l'appellation de culture générale, bien qu'elles prennent solidement appui sur des travers psychologiques dont l'homme cultivé se trouve peut-être affecté plus souvent que d'autres. Mais, ce que la culture générale n'est pas nous indique dans quelle direction chercher ce qu'elle est.

Au fait, dans l'expression «culture générale », le terme problématique est bien plutôt « générale » que «culture ». Car ce dernier substantif, depuis son acception agricole, renvoie analogiquement au travail de l'homme dont on obtient ainsi, comme de la terre, le maximum possible ; tandis que l'épithète «générale » fomente des ambiguïtés qu'illustrent bien les deux conceptions précitées : le vernis culturel, en un certain sens, permet généralement de faire belle figure en société, et la multiplicité des informations comprises assure à peu près aussi généralement qu'on saura se tirer d'affaire. Or, le hasard seul ne peut rendre compte de ces déformations du rôle social et de la fonction pratique de la culture générale : la nature même de la réalité en cause y a aussi sa part. À quoi tient donc le caractère général ici en question?

Il tient, semble-t-il, à l'essence même de ses référents, savoir : ce qui, eu égard au développement humain, est universellement nécessaire, universellement utile et universellement soubaitable. Partant, la culture générale revêt trois significations intimement reliées: une signification fondamentale, une signification instrumentale et une signification téléologique, cha- 
cun de ces termes étant reçu dans son acception stricte. Qu'est-ce à dire au juste? Globalement, cela implique que la culture générale, au sens actif de l'expression, est telle d'abord en ce qu'elle promeut l'épanouissement du corps et de l'esprit, les deux bases faute desquelles tout développement ultérieur, en quelque domaine que ce soit, risque fort d'être boiteux, peut-être même impossible : à ce niveau, la culture générale fait œuvre fondamentale puisqu'elle met en place des conditions de possibilité ; ensuite, en ce qu'elle tend à doter le sujet d'une méthode grâce à laquelle il pourra juger formellement, ce formalisme ouvrant précisément la voie à la généralité dans la mesure où il est moins lié au contenu de la réalité à juger qu'à la manière dont elle se présente, qu'il s'agisse au reste de situations concrètes ou de problèmes théoriques : de ce point de vue, la culture générale fait œuvre instrumentale puisqu'elle fournit un moyen aussi éclairé que possible d'appréhender, d'apprécier et, si requis, de se prononcer et d'agir non seulement en ces matières où le sujet dispose de connaissances précises mais aussi en celles où, contraint de prendre position, il ne peut compter sur un tel savoir ; enfin, en ce qu'elle vise à libérer la personne de toutes les limites que lui imposent ses faiblesses physiques et spirituelles aussi bien que les contraintes sociales et politiques, sans exception d'aucune sorte, de manière à assurer une autonomie aussi complète que possible : à cet égard, la culture générale fait œuvre téléologique puisqu'elle assigne à l'être humain une fin éminemment désirable qu'elle l'aide d'ailleurs à atteindre dans la mesure où elle est humainement accessible. Globalement, cela implique, d'autre part, que la culture générale, au sens passif de l'expression, est telle en ce que le fondement (corps et esprit), l'instrument (méthode) et le but (libération), respectivement nécessaire, utile et désirable dans tous les cas, donc en général, ont effectivement été exhaussés. Ces trois significations se révèlent très intimement reliées du fait que la première conditionne les deux autres tandis que la dernière donne leur sens aux deux précédentes, la méthode articulant ce double rapport.

À ce stade de l'analyse, il devient impérieux de situer la présente conception de la culture générale dans le cadre global 
de l'éducation : cela permettra d'en voir pleinement la portée et, par là, d'évaluer la fonction qui lui revient, s'il y a lieu, dans la formation de l'homme. Après coup, la question du statut de l'enseignement de la philosophie pourra peut-être se présenter sous une forme plus féconde.

\section{ÉDUCATION ET CULTURE GÉNÉRALE}

Ainsi que Kant l'observe, dès le début de ses Réflexions sur l'éducation, "l'homme est la seule créature qui doive être éduquée "; ce qui signifie, en d'autres termes, que l'humanité même est à conquérir et non la seule compétence professionnelle (ou autre) qui, du reste, ne vient qu'ultérieurement en droit, bien qu'en fait ces objectifs soient plus ou moins simultanément poursuivis.

Sous prétexte donc que les divers lieux d'éducation, tels la famille, l'école, la rue et, de plus en plus, les «mass media ", exploitent toute une gamme de procédés, comme le conditionnement, le dressage (dans certains cas, il faudrait même dire l'élevage), l'imitation, l'initiation, l'apprentissage, l'information et l'enseignement, il ne faudrait confondre l'éducation avec aucune de ces pratiques ni avec leur ensemble. $\grave{A}$ cette condition, la valeur architectonique de l'éducation apparaît d'emblée, car, n'ayant le caractère spécifique d'aucun des éléments susmentionnés, elle se révèle elle-même générale aussi bien dans son processus que dans son résultat. $\grave{A}$ cette condition aussi, la place de la culture générale en éducation apparaît essentielle, c'est-à-dire centrale quant au processus et ultime quant au résultat. Concrètement, les implications d'un tel état de choses abondent au point qu'il est impossible de les passer toutes en revue. Par contre, en reprenant, sous ce nouvel éclairage, les trois significations conférées plus haut à la culture générale, les plus décisives seront mises en lumière.

Le processus pédagogique naît avec l'enfant, qu'on le veuille ou pas. Autrement dit, les données fondamentales réunies chez le nourrisson, savoir : son corps et son esprit, fournissent à l'éducation les seules bases naturelles sur lesquelles elle devra tabler, nonobstant toute considération culturelle. Or, ce corps et cet esprit deviennent très tôt et pour longtemps susceptibles de spécialisations diverses. D'où, le débat ultérieur 
opposant les tenants de la formation spécialisée, «professionnelle » à ceux de la formation générale, «libérale». À ce niveau, la culture générale telle qu'entendue ici propose un développement physique et spirituel non spécialisé rendant possible et viable toute greffe spécialisée jugée souhaitable plus tard. En regard du corps, cela signifie, outre une hygiène physique saine, principalement alimentaire étant données ses conséquences sur le développement cérébral, un apprentissage, par la gymnastique, par exemple, ou la pratique des sports, de la maîtrise du corps, grâce à quoi ce dernier deviendra pour le sujet un collaborateur et non un rival ; or, cette culture physique générale ne pouvant être de brève durée, puisque notamment les derniers cartilages se forment en moyenne entre la vingtième et la vingt-deuxième année, elle ressortit réellement au niveau préuniversitaire aussi bien qu'au jeune âge et y occupe en principe une place dont l'importance n'est plus à démontrer dès qu'on a présent à l'esprit l'état physique des adultes. En regard de l'esprit, par ailleurs, cela suppose une bonification des virtualités tant affectives (sentiment, volonté, etc.) que cognitives (raison, imagination, mémoire, etc.), sous l'égide aussi bien de la famille que de l'école, ce qui aboutit à la préséance relative de la famille en matière affective et à la préséance relative de l'école en matière cognitive, puisque ni l'une ni l'autre ne peut tout faire ; partant, la culture générale propose que l'école, et singulièrement l'école préuniversitaire, travaille au développement non spécialisé des connaissances faute desquelles il devient, sinon impossible, du moins très compliqué d'œuvrer adéquatement et, le temps venu, de se spécialiser sans perdre pour autant la polyvalence caractéristique de l'être humain. La question de savoir quelles disciplines particulières il faudrait mettre à contribution dans cette perspective n'a pas à être traitée ici. Cependant, deux remarques s'imposent. Premièrement, ces connaissances indispensables incluent vraisemblablement au moins celle de sa langue en sa qualité de moyen de communiquer, celle également de son histoire (sociale, politique, économique, etc.) en sa qualité de moyen de se situer, celle enfin de sa raison en sa qualité de moyen de penser. Deuxièmement, la philosophie, à supposer qu'un rôle lui revienne en ce domaine, devrait apparemment se consacrer surtout, quoique non exclusivement, au chapitre de la raison ; toutefois, même dans cette hypothèse, elle ne pourrait assumer 
seule la responsabilité de cultiver un champ aussi vaste : la science et l'art, en effet, y ont sans doute un rôle original et irremplaçable à jouer. Mais, c'est plus loin qu'on traitera spécifiquement de ce sujet.

Si la signification fondamentale de la culture générale a permis d'identifier la nécessité de la non-spécialisation de l'éducation au sens kantien du terme, - ce qui n'exclut évidemment pas la formation spécialisée qui ressortirait alors, suivant les cas, à l'instruction technique, à l'apprentissage qualifié, à l'initiation professionnelle, au stage industriel, etc., se déroulant tantôt à l'école, tantôt dans un lieu de travail, la signification instrumentale de la culture générale, quant à elle, permet de clarifier les relations entre formation et information. On sait que ce problème n'est pas théorique : la surcharge des programmes, dénoncée par les étudiants comme par les enseignants, en fournit une illustration souvent dramatique; et les réclamations de certains, désireux de supprimer les études dites inutiles, affrontent depuis longtemps les demandes de certains autres, soucieux de retarder une spécialisation. à leurs yeux précoce et inefficace au profit d'un enseignement de culture devant combler de lourdes carences. À ce sujet, la culture générale propose qu'on préfere clairement la formation à l'information en vertu de la non-spécialisation privilégiée pour les motifs susdits et en vue de favoriser l'accès du sujet à cet instrument en tout cas utile : la méthode. Mais il ne faut pas donner n'importe quel sens à cette proposition. L'éducation, en effet, est un tout où l'on peut cerres distinguer formation et information mais sans pouvoir les séparer de fait. Car la formation pure, dégagée de tout contenu, n'apparaît manifestement pas réaliste au stade de formation étudiée ici; au surplus, le devient-elle jamais? Inversement, les informations nues, prétendument objectives, n'ont aucune valeur éducative en elles-mêmes à moins qu'on ne tienne à considérer le Roquentin de La nausée comme un idéal digne d'émulation. Partant, la position de la culture générale se caractérise en ceci qu'elle tient à ces informations qui favorisent la formation, c'est-à-dire, dans le présent contexte, l'accès à la méthode telle qu'antérieurement définie. Mais, devant la profusion des informations disponibles, lesquelles 
choisir ? et selon quel modèle pédagogique y recourir ? Deux critères orientent ici la sélection. D'abord, tout ce qui est trop spécialisé doit être laissé de côté, le «trop » se mesurant pratiquement à l'aune des connaissances préalables à l'intelligence des informations concernées et à celle de leur utilité technique sur le plan professionnel (cela, bien sûr, sans préjudice de cette partie de la formation préuniversitaire qui vise soit à préparer aux métiers, soit à paver la voie aux études supérieures : mais cette partie, précisément, n'assume pas en tant que telle la formation générale de l'individu). Ensuite, il faut privilégier les modalités d'information dont la teneur permet à l'étudiant des exercices variés et incomparables grâce auxquels il pourra accéder à la méthode par opposition à celles dont la teneur mène à l'enregistrement passif de données, à ce qu'on a coutume d'appeler le «bourrage de crâne » (cela, bien sûr, sans préjudice des renseignements-repères que la mémoire doit forcément emmagasiner pour s'acquitter de sa fonction d'appoint). Donc, un double critère: matériel, d'abord, qui indique ce qu'on doit ou non enseigner ; formel, ensuite, qui fixe la manière dont on doit ou pas procéder.

Un contenu et une pédagogie ainsi constitués découlent d'ailleurs logiquement de la signification instrumentale de la culture générale. En effet, l'acquisition de la méthode, au sens formel du mot, n'est possible que si l'on évite de verser dans l'automatisme et le mimétisme. Or, un contenu surspécialisé qui engendre chez l'étudiant le sentiment d'être dépassé engendre aussi le plus souvent des comportements intellectuels standardisés et incompris retenus à seule raison de leur efficacité présumée ; et une pédagogie de la passivité pousse le sujet assez inévitablement au calque bête, à la répétition à peine masquée et visiblement stérile, vu l'impossibilité d'exercices laissant une certaine marge de mancuvre. Bannir l'automatisme et le mimétisme, c'est acculer le sujet à l'ingéniosité et, par là, rendre possible l'accès à la méthode, en principe applicable en toute circonstance.

Le refus de la spécialisation et la suprématie de la formation prônés par la culture générale en font à coup sûr une pièce centrale du processus éducatif puisque aussi bien les choix pédagogiques en dépendent tous à un titre ou à un autre. 
Il reste donc maintenant à préciser en quoi la culture générale occupe une place ultime quant au résultat recherché par l'éducation, ce que l'examen de sa signification téléogique permet assez bien.

L'être humain se trouve marqué au coin de limites inhérentes, pour ainsi dire par nature, aux capacités brutes dont il dispose. À titre d'exemple, il suffit de noter que le corps, pourtant capable de bien des prouesses, ne peut, sauf exception, s'y livrer spontanément; ainsi, la main du pianiste n'est pas apte d'emblée à l'exécution des arpèges ou trilles que requiert néanmoins l'interprétation de nombreuses auvres musicales. Et, du côté de l'esprit, il en va de même; à preuve: on ne domine pas sa langue du fait unique qu'elle est sienne : au contraire, qui tente de formuler sa pensée, par écrit ou verbalement, découvre très tôt les obstacles multiples qu'elle lui réserve et ressent plus que tout l'insatisfaction que procure une expression inadéquate de soi, voire l'humiliation qu'entraîne la prise de conscience de ses pauvres possibilités. Or, étant donnée cette situation, la fin de libération que poursuit la culture générale déploie tout son sens, car, à cet égard, elle consiste justement et autant que faire se peut à délivrer l'homme, corps et esprit, des résistances dont il est victime. En ce sens, la signification téléologique de la culture générale constitue donc effectivement le résultat ultime auquel peut atteindre l'éducation, puisque là où point la liberté point aussi l'autonomie, c'est-à-dire le temps où s'impose l'interruption des activités pédagogiques comme telles que le sujet et la vie sont désormais à même de relayer. "Le maître doit apprendre à se passer du maitre ": une telle expression n'a de sens que dans cette perspective ou, alors, elle est oiseuse. Cela posé comme cible, comment y parvenir? La culture générale soumer ici que le moyen à mettre en cuvre réside dans la gratuité parce qu'elle fonde la possibilité du transfert. Assertion énorme qui exige des explications !

La complexité extrême du problème du transfert n'est plus à démontrer. Depuis les travaux de Thorndike et Woodworth (1901) jusqu’à nos jours, les expériences et les conclusions de toute nature se sont multipliées, certaines mettant en doute la possibilité même du transfert, la plupart 
en reconnaissant l'existence mais ne s'entendant guère sur sa nature (transfert de structures - externes ou fonctionnelles ou transfert de comportements; transferts de diffusion, d'adaptation ou de transposition ; transferts rigides ou plastiques, immédiats ou acquis, positifs ou négatifs ; etc.). Or, comme le transfert fonde l'apprentissage (Fergusson, 1954) et, par ce biais, une large part de l'éducation, la question se complique à souhait du fait que les théories de l'apprentissage abondent, qu'il s'agisse d'apprentissage perceptif, moteur, verbal ou intellectuel : qu'on songe ici aux théories du réflexe conditionné (Pavlov, Watson, ....), du renforcement des connexions (Hull, Spence, ....), de l'expectation (Tolman, Guillaume, ....), de l'équilibration (Piaget, Nuttin, ...), etc.. D'ailleurs, si les psychologues admettent que l'apprentissage joue un rôle notamment dans l'activité cognitive, ils avouent qu'il est mal connu et, de toute façon, contesté (Le Ny, 1968), qu'au surplus le transfert qui est à sa base ne peut être étudié systématiquement dans « des perspectives trop complexes comme celles de l'éducation »(Oléron, 1968). Au bilan, si certaines lois semblent acquises, telles celles de répétition et de contiguïté temporelle des exercices, le problème de l'apprentissage demeure largement ouvert comme d'ailleurs la question de savoir quelles fonctions le transfert y assume. Toutefois, la nature du transfert fait l'objet d'un consensus relatif : il y a transfert "dans tous les cas où une activité modifie d'une façon quelconque par facilitation ou par interférence celle qui la suit $\gg\left(\right.$ Oléron, 1968) ${ }^{1}$. Or, c'est évidemment l'aspect de facilitation qui, dans le transfert,

1. Sur toutes ces questions, voir, en particulier, Fekciusson, G.A., "On learning and human ability ", Canadian Journal of Psychology, 1954, VHI, p. 95-112 ; Ginll.ALme, P., La formation des hahitudes, Paris, P.U.F., 1947 ; Hul., C. L., A bebavior system, New Haven, Yale University Press, 1952 ; LE NY, J.-F., "Apprentissage ", Encyclopadia Universalis, Paris, E. U. France, 1968 , II, p. 173 b-175 c ; NUTTlN, J., Tâcbe, réulsite et échec, Louvain, Publications Universitaires, 1953 ; OlEkon, G., Le transfert in Frassie, P. et $\mathrm{P}_{\mathrm{A} A \mathrm{E}} \mathrm{T}, \mathrm{J}$. (Éd.), Traité de psychologie expérimentale, Paris, P.U.F., $2^{c}$ éd., 1968, IV, p. 119-181; PAVIOV, I. P., Conditioned reflexes, London, University Press, 1927 ; Placiet, J., Apprentissage et connaissance in Études dépistémologie génétique, Paris, P.U.F., 1959, VII, p. 21-67 et X, p. 159-188; SPENCE, K., Behatior theory and learning, New York, Prentice Hall, 1960 ; THORndike, E. L. et WoODworTH, R. S., "The influence of improvement in one mental function upon the efficiency of other functions", Psychological Review, 1901, VIII, p. 247-261, 384-395 et 553-564 ; TOLMAN, E. C., Principles of purposive bebavior in $\mathrm{K}(\mathrm{CH}, \mathrm{S}$. (Ed.), Psychology. A study of a science, New York, McGraw Hill, 1959, II, p. 92-157; WATSON, J. B., Psychology from the standpoint of a behaviorist, Philadelphia, Lippincort, 1919 
intéresse l'éducation puisqu'elle cherche à faire progresser le sujet et non à lui nuire. Et c'est précisément pour cette raison de facilitation que la culture générale privilégie la gratuité des exercices.

En effet, un exercice désintéressé, c'est-à-dire ne visant pas à court terme une performance professionnelle précise, ne peut avoir qu'un résultat général: rendre l'organe exercé disponible à toutes fins utiles par opposition aux exercices intéressés qui aboutissent forcément aux seuls résultats recherchés et entravent inévitablement l'acquisition d'autres habiletés. Ainsi, l'exercice physique, par exemple la gymnastique, qui ne vise d'autre fin que la mise en disponibilité du corps porte-t $t$-il des fruits transférables, tels l'endurance, la souplesse et le contrôle; alors que l'exercice physique ordonné à une fin particulière, disons: la performance cycliste, développe fort inégalement l'appareil physique, entraîne en particulier une déformation typique de la colonne vertébrale et handicape ainsi l'individu lorsque vient le moment de s'adonner à un autre type d'activité corporelle. Mutatis mutandis, la même constatation s'applique à l'esprit; des activités intellectuelles intéressées, ordonnées à un savoir technique risquent au mieux de faire advenir, avec une compétence restreinte, une déformation professionnelle qui, de toute manière, vient toujours trop tôt ; alors que des exercices gratuits assouplissent les facultés, ce qui servira toujours, apprennent au sujet de quoi elles sont capables, ce qui inspire une confiance constamment requise, lui enseignent quelles en sont les limites, ce qui risque de diminuer les présomptions invariablement fatales. Pour tout dire, mieux vaut étudier les mathématiques et la langue pour elles-mêmes que de s'en tenir aux techniques de la comptabilité ou aux tournures de la langue des affaires: celles-ci, en effet, ne sont surtout pas généralisables tandis que celles-là leur ouvrent la voie, comme à bien d'autres connaissances particulières d'ailleurs, sans compter qu'elles forcent le rodage de dispositions continuellement précieuses, telles la rigueur et la nuance. En un mot, elles rendent l'esprit disponible, c'est-à-dire capable de s'adapter parce que libre d'cillères contraignantes dans la mesure, cela va de soi, où c'est humainement réalisable et où l'enseignement de culture ne tourne pas lui-même à l'exposé universitaire, donc spécialisé. 
À la lumière de la problématique esquissée jusqu'à présent, il devient possible, jusqu'à un certain point, de formuler quelques conclusions relatives à l'apport de l'enseignement de la philosophie à la culture générale.

\section{PHILOSOPHIE ET CULTURE GÉNÉRALE}

Il y a d'abord une évidence qu'il ne faut pas craindre de signaler: l'inculture ne tue pas ! Ce qui implique, à parler absolument, que la vie ne requiert pas davantage un enseignement de la philosophie qu'une éducation de culture. La première conséquence de ce truisme est de dédramatiser une question trop souvent plongée dans un tourbillon de discussions plus passionnées qu'éclairantes. Sa deuxième conséquence est de faire voir, dans la foulée des analyses précédentes, que, parce qu'objet de choix et porteuse de valeur, la culture générale - et, peut-être, la philosophie - revêt assurément en éducation une importance décisive par rapport aux caractères spécifiques de l'homme: en ces matières, le prix qu'on attache à ses préférences est à proportion de la liberté qu'on y investit. Ensuite, il faut rappeler que la culture générale constitue un tout auquel, en droit du moins, chaque discipline doit apporter sa contribution ; la formation scientifique, pour ne citer qu'un cas, est ausşi une formation morale dans la mesure où elle passe forcément par l'apprentissage de l'honnêteté avec les faits : en ces matières, il n'existe pas de chasses gardées. D'où, aucun enseignement ne peut s'arroger le pouvoir exclusif d'assurer la culture générale ; d'où, encore, aucun enseignement ne peut prétendre faire une chose et une seule. Si bien que, dans l'éventualité où la philosophie remplirait un rôle même indispensable et prioritaire du point de vue de la culture générale, elle ne saurait l'assumer seule et elle ne saurait assumer que lui : en ces matières, l'impérialisme et l'exclusivisme ne siéent point. Enfin, on ne trouve pas chez l'homme de faculté particulière dont le développement reviendrait à la culture générale, voire à la philosophie, à l'instar de la faculté de mémoire dont le développement ressortit à des pratiques identifiables aux résultats mesurables. De sorte qu'exiger, en l'occurrence, de la culture générale et de la philosophie, s'il y a lieu, qu'elles produisent la fonction 
humaine particulière cultivable par leur office représente une prétention irrecevable au nom de laquelle nul ne peut leur refuser leurs quartiers de noblesse ; de sorte, également, que ni la culture générale ni la philosophie, le cas échéant, ne doivent restreindre leur champ d'intervention, dussent-elles etre terrifiées par la tâche : en ces matières, céder du terrain équivaut à perdre son sens.

Voilà donc quelques coordonnées passablement claires qui, s'appliquant à la culture générale, vaudraient aussi pour l'enseignement de la philosophie si une place lui revenait dans ce vaste domaine. Mais, au fait, une telle place lui revientelle? À cela, il faut répondre deux choses. Premièrement, non, une telle place n'a pas à lui revenir, puisque le cours de philosophie n'est pas nécessaire, stricto sensu, à la culture générale; à la vérité, non seulement d'autres disciplines peuvent y mener par d'autres moyens, mais en réalité des peuples entiers qui n'en sont pourtant pas dépourvus ne dispensent aucun enseignement préuniversitaire obligatoire de la philosophie : le cas d'une majorité de lander allemands en fournit une illustration d'autant plus frappante que la philosophie elle-même y fleurit particulièrement bien. Deuxièmement, oui, une telle place peut devoir lui revenir, puisqu'en droit la philosophie répond aux exigences sous-tendues par les trois significations de la culture générale au même titre que plusieurs autres disciplines et qu'en cette qualité elle mérite qu'on retienne sa candidature ni moins ni plus que celle de ces dernières. Il faut bien voir ici qu'il s'agit d'un choix, justifiable certes, mais d'un choix difficile néanmoins en ce qu'il porte sur des réalités relativement équivalentes du point de vue qui prévaut ici.

Et, d'abord, la philosophie répond à la signification fondamentale de la culture générale : elle promeut effectivement l'épanouissement de l'esprit en lui fournissant un point de vue original sur le réel, en lui ouvrant des perspectives le plus souvent insoupçonnées par ailleurs, en lui posant quelques interrogations insolites, somme toute en lui faisant quitter les sentiers habituels à la faveur d'une quête radicale plutôt vouée à bouleverser, à transformer qu'à apprendre. Elle répond, ensuite, à sa signification instrumentale: elle favorise forcé- 
ment l'acquisition d'une méthode en confrontant le sujet à des problèmes vitaux qu'il lui faut tenter de résoudre, en attirant explicitement son attention sur des méthodes explicitement présentées pour leur valeur paradigmatique, en l'amenant à se situer à un niveau de principe où ses mécanismes usuels perdent leur efficace, en un mot en le plaçant sur un terrain où ses pseudo-méthodes échoueront qu'il lui faudra bien remplacer. Elle répond, enfin, à sa signification téléologique : elle provoque la libération de l'esprit en ouvrant des horizons que la plupart n'ont pas pris en compte jusque-là, en mettant en cause des positions captivantes reçues par la majorité comme allant de soi, en exploitant l'intelligence discursive qu'elle fournit à chacun l'opportunité de structurer, au bilan en avivant les faiblesses d'un esprit encore en gestation et en les lui rendant suffisamment intolérables pour qu'il veuille enfin s'en départir. Voilà ce qu'en droit la philosophie apporte à la culture générale et que d'autres disciplines pourraient lui apporter en procédant autrement; voilà pourquoi, en droit, le cours de philosophie pourrait être choisi en regard de la culture générale. Maintenant, deux questions se posent : en fait, qu'en est-il ? et : n'y a-t-il pas quelque valeur typique de la philosophie en vertu de laquelle elle devrait être privilégiée par rapport à d'autres matières qu'il serait pourtant parfaitement sensé de retenir?

En fait, l'enseignement de la philosophie n'a de chance de remplir son office que si l'on respecte certaines conditions élémentaires trop fréquemment négligées. Au premier chef, il importe que le climat global d'une maison d'éducation n'aille pas à l'encontre d'un enseignement de culture, car ni la philosophie ni aucune autre discipline ne peuvent, seules, effectuer tout le travail pertinent; donc laisser à la philosophie la portion congrue, la noyer dans une masse de cours plus ou moins techniques, l'isoler dans sa gratuité équivaut, à peu près, du point de vue du rendement, à la supprimer. D'autre part, de par sa nature même et de par les moyens qu'elle met en œuvre, la formation philosophique ne peut, pour des raisons évidentes, s'adresser à n'importe quel groupe d'âge ; au-dessous d'un certain seuil, l'appellation "philosophie » risque fort de recouvrir n'importe quoi sauf ce qu'elle devrait, avec 
toutes les implications qu'on soupçonne. Par ailleurs, et pour des raisons analogues, elle ne peut s'adresser de la même façon à n'importe quel auditoire; or, il ne s'agit pas ici d'élitisme peu ou prou bourgeois, mais d'une constatation découlant tout uniment du fait qu'on ne peut imposer profitablement à quiconque un style d'éducation plutôt qu'un autre; notre société l'a bien compris qui ne contraint même pas les plus doués aux études postsecondaires; et, pourtant, cette même société impose à tous les étudiants préuniversitaires des cours communs de philosophie, alors qu'ils sont nombreux ceux qui poursuivent leurs études à ce niveau parce que c'est le seul moyen d'accès au métier qu'ils désirent exercer ou parce que le marché du travail les oblige à un supplément de formation technique non indispensable en soi, mais avantageux étant donné le surplus de travailleurs en situation de chômage chronique; au nom de la démocratisation de l'éducation, on force des personnes à des études qu'elles éviteraient peut-être alors même qu'elles en connaîtraient la pleine valeur. Enfin, la culture générale, et spécialement celle qui passe par la philosophie, requiert du temps et du temps employé à bon escient, car elle repose, entre autres bases, sur une osmose de longue haleine; à vouloir faire vite ou court, en ce cas, on risque de ne rien faire de valable, surtout si, de surcroît, on procède selon des horaires accablants pour l'étudiant, allant carrément à l'encontre de toutes les connaissances acquises à propos des durées d'attention dont un être humain, et adolescent encore, est capable. Mais ces problèmes de fait se posent aussi bien aux autres disciplines de culture générale qu'à la pinilosophie. Ce qui renvoie à l'aspect de droit qui veut savoir si, oui ou non, il y a lieu de privilégier la philosophie.

En droit, il existe, semble-t-il, une valeur typique de la philosophie qui justifie, sans toutefois l'obliger, qu'on lui octroie un statut préférentiel eu égard à la culture générale : il s'agit de sa qualité de foyer de synthèse critique. Rien, en effet, n'échappe à la réflexion philosophique: de l'être à la sexualité, en passant par l'art, la politique, le passé, la mort, tout a été ou est passible d'être traité philosophiquement. Synthétique donc, la philosophie focalise les thèmes abordés en un point particulier, le seul probablement qui n'ait jamais 
varié dans toute son histoire : la question, c'est-à-dire l'examen critique par lequel on trouve sens, on donne sens ou on refuse sens à la réalité. D'autres disciplines, bien sûr, remplissent une fonction critique : la science politique, par exemple, qui s'en tient de moins en moins exclusivement à la description et à l'explication ; mais y a-t-il une science politique de la mort? ou de l'art? La synthèse ici ne paraît guère réalisable. D'autres disciplines, dira-t-on, sent fort synthétiques, et l'on en voudra peut-être l'histoire pour preuve. Soit! Mais la critique y trouvera-t-elle son compte? Pourra-t-elle jamais, par exemple, tenter de répondre aux trois célèbres questions de Kant? D'ailleurs, ne demande-t-elle pas elle-même à l'historien des sciences d'être au moins vaguement homme de science ? et à celui de la philosophie d'être quelque peu philosophe?

Foyer de synthèse critique : la philosophie ainsi conçue ne constituerait-elle pas, assez singulièrement au reste, un facteur de libération économique, sociale et politique? d'émancipation scientifique, esthétique, morale et, pourquoi pas, religieuse ? Mais, au fond, la liberté semble si lourde à porter et paraît compter tant d'aléas qu'en somme elle joue peut-être aussi bien contre la philosophie que pour elle. Et, au demeurant, si l'histoire nous enseigne que la philosophie ne sait pas mourir, elle nous apprend, en revanche, que l'enseignement de la philosophie n'est jamais menacé que sous de mauvais prétextes. Après tout, les «bons prétextes " tiennent peut-être de l'inavouable...

Collège Ahuntsic

Montréal 